\title{
First 10Gb/s Transmission with radiation-hardened Silicon Photonic Mach-Zehnder Modulators in a Full Transmission System
}

\author{
Marc Schneider ${ }^{*}$, Yunlong Zhang, Djorn Karnick, Lars Eisenblätter, Thomas \\ Kühner, Marc Weber \\ Karlsruhe Institute of Technology (KIT), Institute for Data Processing and Electronics (IPE) \\ Kaiserstr. 12, 76131 Karlsruhe, Germany \\ E-mail: marc.schneider@kit.edu, zhang.yunlong@partner.kit.edu, \\ djorn.karnick@partner.kit.edu, lars.eisenblaetter@kit.edu, \\ thomas.kuehner@kit.edu, marc.weber@kit.edu
}

\begin{abstract}
We present standard and radiation-hardened pn depletion-type Mach-Zehnder modulators (MZM) to be used in high-bandwidth, fully-integrated wavelength division multiplexing (WDM) transmitter designs, aiming to upgrade the optical data transmission of future detector systems. A detailed characterization of the modulators with respect to modulation efficiency and $\mathrm{RF}$ response was carried out. Based on this work, a first optical link with a data rate of $11.3 \mathrm{~Gb} / \mathrm{s}$ was set up.
\end{abstract}

Topical Workshop on Electronics for Particle Physics (TWEPP2019)

2 - 6 September 2019

Santiago de Compostela, Spain

\section{*Speaker}




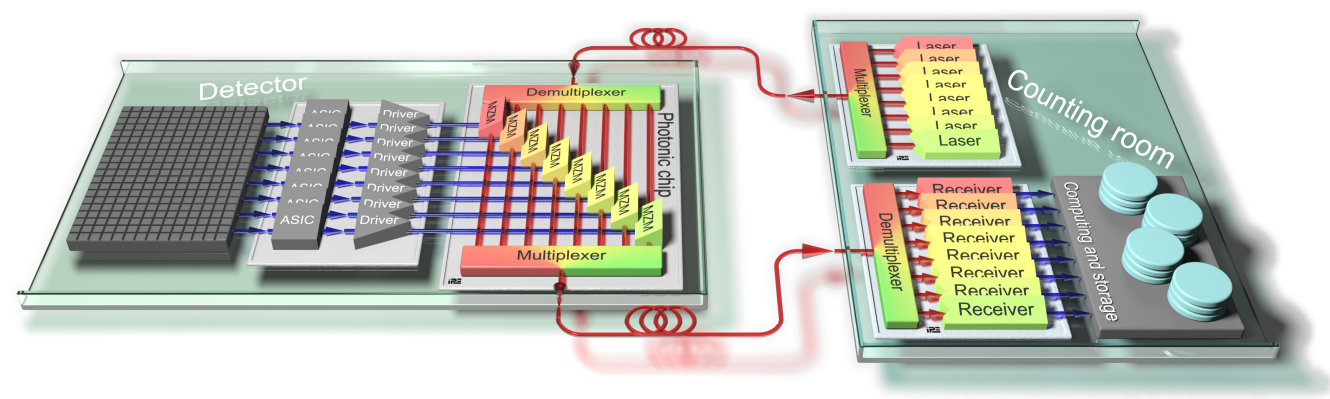

Figure 1: Schematic view of a WDM system concept for detector instrumentation.

\section{Introduction}

The number of detector read-out channels as well as the data rate of each individual channel are rapidly increasing in detector instrumentation. Future high energy physics (HEP) and photon science detector systems will have many millions or even billions of channels [1]. Even with data reduction and compression, a large amount of data has to be transferred to the counting room. Optical links used in current large-scale detector systems employ directly modulated laser diode transmitter units, where each is connected by an individual optical fiber to an offdetector receiver. The transmitter consists of vertical cavity surface emitting lasers (VCSEL) and a data rate of $10 \mathrm{Gbit} / \mathrm{s}$ per fiber is achieved in typical HEP applications. The most viable way to cope with an ever increasing data throughput is adding more and more fibers at the price of increased mass and volume. But more importantly, VCSELs will not be able to withstand the expected radiation levels in the innermost regions of future HEP experiments [2]-[4].

Our data transmission system (Figure 1), based on silicon photonics and wavelength division multiplexing (WDM), can resolve the challenge of transmission bandwidth [5],[6] and, in all probability, radiation hardness. Lasers located outside the detector generate multiple optical carriers, which are combined by an optical multiplexer and transmitted over a common singlemode glass fiber (SMF) to a transmitter unit on a photonic chip inside the detector. The optical carriers are demultiplexed on-chip and fed into distinct Mach-Zehnder modulators (MZMs). The electrical signals from the sensor elements are pre-processed by custom read-out ASICs, mapped to available transmitter channels, and amplified to drive the electro-optic MZMs. The modulated optical carriers are multiplexed on-chip and transmitted through a second SMF to the counting room. There, the signals are demultiplexed again and routed to individual receivers. The photonic chip inside the detector volume will be completely customized and tailored to the requirements, while the off-detector equipment is assembled from commercial off-the-shelf components to minimize development costs.

In this paper we present our measurements on a full single channel data transmission system with a data rate of $11.3 \mathrm{Gbit} / \mathrm{s}$ using different MZMs designs to prove their suitability for a complete WDM system.

\section{Mach-Zehnder Modulators}

High-performance photonic modulators are key components for active silicon photonics, and carrier-depletion phase modulators are well suited for WDM applications. The MZMs consist of two pn-phase shifters, one in each arm of a Mach-Zehnder interferometer (Figure 2 a), 
implemented by a horizontal pn-junction integrated in a waveguide and operated with reverse biasing (Figure 3). The number of free carriers determines the refractive index which can be thus changed by varying the voltage across the junction. Hence, the phase of light passing through the structure is manipulated. If the electrical signals differ, the refractive indices of the phase shifters and hence the optical phases at their outputs differ and the light intensity of the combiner output is reduced due to destructive interference in the output combiner. The downside of this simple construction is that a proper working point has to be set by applying a reverse voltage to one of the phase shifters to bring the optical output intensity to the quadrature point, the midpoint between maximum and minimum intensity, which reduces the efficiency of the affected phase shifter.

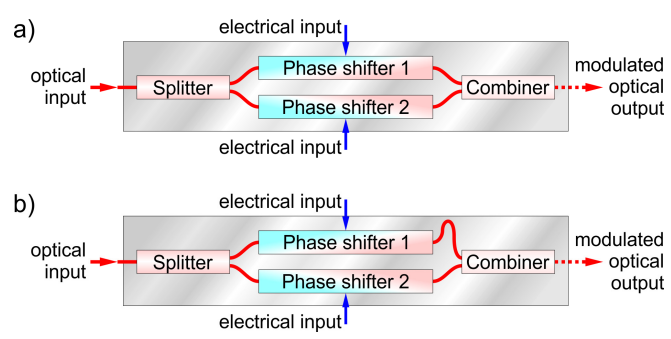

Figure 2: Schematic of a) symmetric and b) asymmetric MZMs

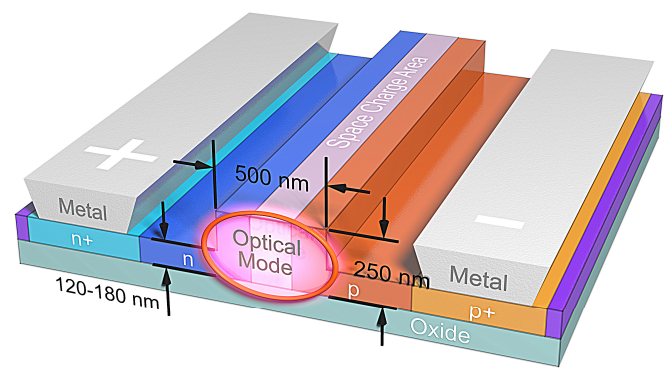

Figure 3: Schematic of a phase shifter with a horizontal pn-junction.

In order to facilitate modulator characterization, the MZM can be designed asymmetrically (Figure 2 b), i.e. with different arm lengths. Then, the operating wavelength is an additional degree of freedom in the selection of the operating point. Thus an appropriate operating point can be adjusted by varying the operating wavelength.

On our photonic chip we implemented both symmetric and asymmetric MZMs. While the asymmetric MZMs are individual devices, the symmetric MZMs are part of an integrated, 4-channel WDM transmitter system. The system modulators are equipped with dual optical input and output ports. Thus, they can be characterized individually apart from operating as part of the WDM transmitter. For both types the electrical connections are thin doped silicon slabs on the sides of the waveguide rib shown in Figure 3. The slab thickness of these standard modulators is $120 \mathrm{~nm}$, providing a good confinement of the optical mode in the waveguide rib. Research at CERN [3],[4] showed that such a phase shifter can be made much more tolerant against radiation, if the slab thickness is increased significantly. The downside is a less confined optical mode and a slightly degraded modulator performance. Our radiation-hardened MZMs feature a $50 \%$ higher slab thickness of $180 \mathrm{~nm}$, but radiation hardness has still to be shown. The phase shifters of all discussed MZMs are $3 \mathrm{~mm}$ long.

Fabrication was conducted by IMS Chips, Stuttgart, on a $250 \mathrm{~nm}$ SOI platform in a full custom process with three etch depths. Electron beam lithography was used for all photonic structures with feature sizes up to $4 \mathrm{~mm}$ and down to $160 \mathrm{~nm}$, while the metallization was structured by UV lithography. A $\mathrm{SiO}_{2}$ top layer, which is not shown in Figure 3, acts as passivation and upper cladding for the optical waveguides. Further details can be found in [6].

\section{Measurement Setup}

We measured the data transmission performance of the different MZMs described above with a laboratory setup representing a full data transmission system (Figure 4). A pseudo-ran- 


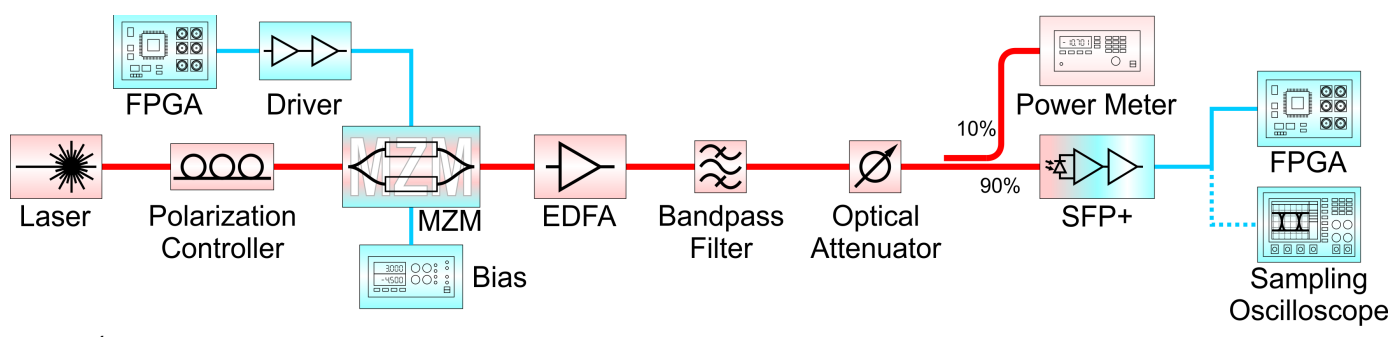

Figure 4: Schematic view of the used system measurement setup.

dom bit sequence (PRBS) with a length of $2^{7}-1$ bits and a rate of $11.3 \mathrm{Gbit} / \mathrm{s}$ was generated by an Altera Stratix V GX Transceiver Signal Integrity Development board. The electrical setup can be seen in [6]. The optical carrier was generated by a tunable laser at a power of $6 \mathrm{dBm}$ and a wavelength around $1550 \mathrm{~nm}$. Correct polarization for efficient coupling was maintained by a polarization controller. The optical output of the modulator was fed into an optical amplifier (EDFA). An optical bandpass filter removed spectral components from spontaneous emission of the EDFA. With a variable optical attenuator the average optical power was set to the desired value, which could be measured by a power meter connected to the $10 \%$ branch of a 90:10 splitter. The main part of the signal was fed into the receiver side of a commercial SFP+-module (Fiberstore SFP-10GER-55). The module could be connected to a sampling oscilloscope for setup or to the FPGA board for bit error rate measurements.

\section{Results}

We measured the bit error rate (BER) of different MZMs at a data rate of $11.3 \mathrm{Gbit} / \mathrm{s}$ while varying the amplitude of the electrical driving signal for the modulators. The EDFA and the optical attenuator were set to values to achieve an optical power of $0 \mathrm{dBm}$ at the receiver and at least $6 \times 10^{12}$ bits were transmitted for each measurement.

Figure 5 shows the BER measurements for a standard MZM with $120 \mathrm{~nm}$ thick slabs and for a radiation-hardened MZM with $180 \mathrm{~nm}$ thick slabs, both asymmetric and $3 \mathrm{~mm}$ long. The driving amplitude was varied from $3.15 \mathrm{~V}_{\mathrm{PP}}$ to $0.88 \mathrm{~V}_{\mathrm{PP}}$. For both modulators the BER is below $10^{-12}$ for amplitudes above $1.65 \mathrm{~V}_{\mathrm{PP}}$ (indicated by arrows in the figure). For lower voltages the BER rises significantly and below $0.9 \mathrm{~V}_{\mathrm{PP}}$ no valid bits were recognized anymore by the FPGA.

The same measurements were conducted for the four symmetric, $3 \mathrm{~mm}$ long MZMs of a WDM system with $120 \mathrm{~nm}$ slab thickness and the results are shown in Figure 6. Two modulators, namely channel 1 and 4, show a similar behavior to the asymmetric MZMs. Channel 1 shows the best results with a BER below $10^{-12}$ for a modulation amplitude above $1.3 \mathrm{~V}_{\mathrm{Pp}}$. Here the rather low required biasing voltages come into play. Channel 4, requiring higher biasing, shows a low BER for modulation amplitudes above $1.3 \mathrm{~V}_{\mathrm{PP}}$, but a long plateau with a BER slightly above $10^{-12}$. The BER was smaller than

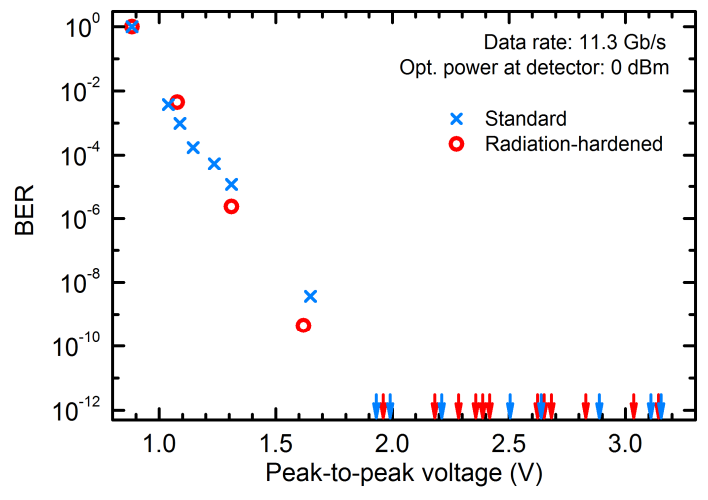

Figure 5: BER measurements for different driving amplitudes for asymmetric standard and radiation-hardened MZMs. 
$10^{-12}$ for amplitudes above $2.75 \mathrm{~V}_{\mathrm{PP}}$. Channels 2 and 3 show a very high BER for all driving amplitudes and a much flatter characteristic. The reason for the high BER is the small extinction ratio of the modulators, which is a consequence of the inconvenient operating points that can be selected by biasing. This, in turn, is due to small variances of the MZM arm lengths ratio, although designed identically.

\section{Conclusions and Outlook}

We presented the transmission of an

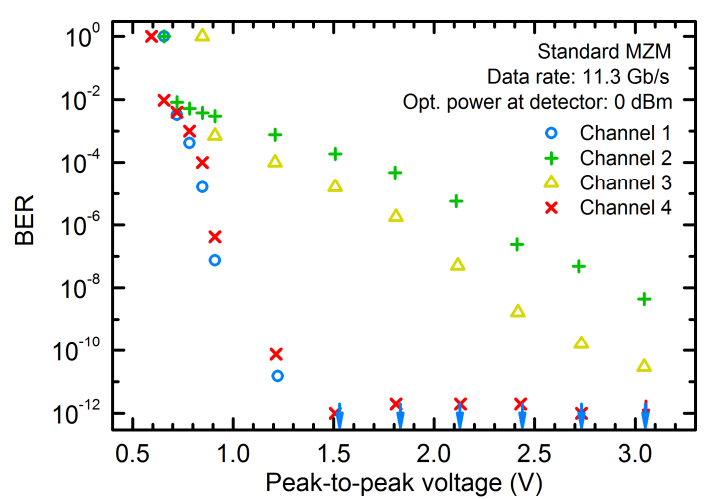

Figure 6: BER measurements for different driving amplitudes for symmetric standard MZMs.

$11.3 \mathrm{Gbit} / \mathrm{s}$ data signal with both standard and radiation-hardened modulator designs with phase shifter lengths of $3 \mathrm{~mm}$. In an optimum operating point, error-free transmission is demonstrated for an electrical driver signal amplitude down to $1.65 \mathrm{~V}_{\mathrm{PP}}$. This low driving voltage enables the integration of the modulators with small-structure CMOS ASICs. In order to improve the performance especially of the symmetric MZM, a wider range to adjust the operating point is required. In a future modulator design, this can be achieved by inserting thermal phase shifters in the interferometer arms.

\section{Acknowledgements}

This research is supported by the program Matter and Technology of the Helmholtz Association, Germany and the China Scholarship Council (CSC), China.

\section{References}

[1] See, for example: CMS Collaboration, The Phase-2 Upgrade of the CMS Tracker, Technical Design Report, Preparatory Group, CERN-LHCC-2017-009; CMS-TDR-014, 2017.

[2] J. Troska et al., Radiation damage studies of lasers and photodiodes for use in multi-Gb/s optical data links, IEEE Trans. Nucl. Sci. 58 (6), pp. 3103-3110, 2011

[3] M. Zeiler et al., Radiation Damage in Silicon PhotonicMach-Zehnder Modulators and Photodiodes, IEEE Trans. on Nucl. Sci. 64 (11), pp. 2794-2801, 2017.

[4] A. Kraxner et al., Investigation of the Influence of Temperature and Annealing on the Radiation Hardness of Silicon Mach-Zehnder Modulators, IEEE Trans. on Nucl. Sci. 65 (8), pp.1624-1631, 2018.

[5] D. Karnick et al., Optical links for detector instrumentation: on-detector multi-wavelength silicon photonic transmitters, JINST 12, C03078, 2017.

[6] Y. Zhang et al., Key building blocks of a silicon photonic integrated transmitter for future detector instrumentation, JINST 14, P08021, 2019. 